\title{
Hybrid Cargo-Level Tracking System for Logistics
}

\author{
Guang-Hua Yang, Kuang Xu, Victor O.K. Li \\ Department of Electrical and Electronic Engineering \\ The University of Hong Kong \\ Pokfulam Road, Hong Kong, China
}

\begin{abstract}
In this paper, we propose a hybrid cargo-level tracking system for logistics. We highlight the special system requirements, discuss the design issues and identify the design principles. Then we propose an innovative hybrid system. As far as we know, this is the first system that exploits both infrastructure-based and infrastructure-less positioning schemes for practical cargo-level tracking. Compared with existing systems, the proposed system provides a ubiquitous cargo-level tracking solution with enhanced availability, reliability, and lower total costs.
\end{abstract}

\section{INTRODUCTION}

With the growth in global business activities, it becomes essential for the firms to manage the logistics flow and track their cargos. Continuous monitoring and tracking are required for shipments of high value and important cargos such as jewelry, electronic products, legal documents, etc. Although global position system (GPS) is available for global tracking and radio-frequency identification (RFID) is emerging for pallet-level visibility, they represent the two ends of the tracking technology spectrum. The gap still exists for seamless and continuous tracking at the cargo-level for transit from indoors to outdoors, from warehouses to containers.

Cargo-centric tracking with genuine end-to-end continuous tracking and monitoring capability is still in the research stage. Existing tracking systems have not fully addressed the issues of transit over diverse transportation modes and environments. They often overlook the environments during transit and none can monitor the cargo continuously with their single positioning technology. Another problem of existing tracking systems is the high costs for deployment and operation. Existing container-level tracking systems can not be used for cargo-level tracking, due to the large number of objects to be tracked. The industry generally lacks innovative and costeffective devices and applications for the need in cargo-level tracking.

The major objective of this work is to develop the nextgeneration tracking system which supports continuous, ubiquitous cargo-level tracking. The main contributions of this work are three-fold.

- In the context of a cargo-level tracking system, the special system requirements are highlighted, the design issues are discussed, and the design principles are identified.

- An innovative hybrid system is proposed. As far as we know, this is the first work that exploits both

This research is supported in part by the Innovation and Technology Fund of the Hong Kong Special Administrative Region, China, under Grant No. ITP/019/09LP. infrastructure-based and infrastructure-less positioning schemes for a practical cargo-level tracking system.

- Cost-effective tracking devices and a central server for cargo-level tracking applications are developed.

The rest of this paper is organized as follows. In Section II, we introduce the properties of a positioning system. Major existing positioning technologies and systems are also introduced. In Section III, we highlight the special requirements of a cargo-level tracking system, and discuss the design issues involved. In Section IV, we introduce the system design and development in detail. Finally, we conclude in Section V.

\section{Positioning Technologies And Systems}

\section{A. Positioning system properties}

There are many existing positioning systems using various approaches. Usually each approach solves a different problem or supports a different application. When we design a positioning system, we should consider what system parameters are important to the specific application. The major parameters to be considered include:

- Absolute or relative location: An absolute location system, such as GPS, uses a shared reference grid for all objects. In a relative system, each object may have its own frame of reference.

- Distributed or centralized computation: The positioning related calculation may be performed at the tracking device in a distributed way or at a central server.

- Accuracy and precision: For instance, when we say that a positioning system achieves 5 meters accuracy 90 percent of the time, 5 meters denotes the accuracy and 90 percent denotes precision. Generally, we can trade accuracy with precision.

- Availability and coverage: GPS provides global coverage. However it can not work indoors and does not work well in areas with limited view of the sky. These limitations result in an availability of coverage of only $4.5 \%$ of the time of a user's daily life [1]. Generally, improved availability and coverage can be obtained by deploying more infrastructures, but with increased costs.

- Scalability: GPS can serve an unlimited number of receivers worldwide, while some positioning systems, such as those based on Infrared [2], can only work in a preconfigured small area and support very limited number of objects.

- Costs: Costs include costs for deployment, operation and maintenance. Deployment costs include the costs of manufacturing and deploying the infrastructures, tracking 
device, and administration systems. Operation and maintenance costs are incurred to keep the system working properly and smoothly.

- Limitations: Different positioning systems suffer different limitations. For example, GPS can not work indoors and in city canyons; time-difference-of-arrival (TDOA) based system [3] requires perfect synchronization among tracking device and reference nodes.

\section{B. Location techniques and systems, such as GPS}

Based on whether infrastructure is required, existing positioning techniques can be classified into infrastructure-based schemes and infrastructure-less schemes.

1) Infrastructure-based schemes: The Infrastructure-based schemes generally involve estimations of distances or directions of an object with respect to a set of infrastructure anchor nodes. Systems based on time-of-arrival (TOA) [4], TDOA [3], angle-of-arrival (AOA) [5] and radio received signal strength (RSS) are examples.

TOA [4] and TDOA [3] estimate range directly based on timing information. The accuracy of the estimation is usually hampered by additive noise [6] and multi-paths [7]. They also require perfect time synchronization among the object and the anchor nodes, and this is a challenge in a practical system. AOA [5] measurements give the direction to the anchors rather than the range. AOA approaches generally require special hardware with multiple antenna elements, and the requisite extra costs and space. RSS is the received signal strength measured at the receiver. This value is normally reported as an integer number, RSS indicator, or RSSI in brief. RSS measurements are relatively inexpensive. Measurements can be made at each receiver during normal data communications, without additional bandwidth or energy requirements, and are simple to implement in hardware.

Given the range information, a positioning system can determine the position of an object using triangulation, multilateration, or scene analysis techniques. The details of these techniques can be found in [8].

The best-known infrastructure-based location system is GPS. As noted above, although accurate and very effective in open environment, GPS has limited coverage and availability [1]. Other popular infrastructure-based location systems are based on infrared [2], ultrasound [4], or radio frequency (RF) systems such as Bluetooth, WiFi [9] and GSM [10] cellular networks.

The rapid deployment of WiFi infrastructures and popularity of WiFi-enabled devices make WiFi-based positioning attractive. The RADAR project [9] pioneered indoor WiFi positioning. In RADAR, a fingerprinting-based mechanism is employed. The hypothesis is that at a given point, a user may hear different access points (APs) with certain signal strengths; this set of APs and their associated signal strengths represent a fingerprint that is unique to that position. The fingerprint algorithm is performed in two stages. In the first stage, the radio map at different locations is constructed by methods such as wardriving [1]. Then in the second stage, a device performs a scan of its environment and compares the collected information with the obtained radio map to get a matching.
With a high-resolution radio map and an effective matching algorithm, WiFi-based positioning can provide similar or even better accuracy than GPS.

2) Infrastructure-less schemes: Infrastructure-less positioning schemes are generally employed in ad hoc sensor networks, in which the network topology and connectivity information are exploited for positioning. In an infrastructure-less system, there are typically two types of nodes: anchor nodes and blind nodes. Anchor nodes have a priori knowledge of their own positions with respect to some (absolute or relative) coordinate system. Blind nodes have unknown positions and need to be located.

DV (distance vector)-hop approach developed by Niculescu et al. [11] is a representative of infrastructure-less positioning systems. DV-hop starts with all anchors flooding their locations to other nodes in the network. The messages are propagated hop-by-hop and a hop-count is carried in the message. Each node maintains an anchor information table and counts the least number of hops that it is away from an anchor. When an anchor receives a message from another anchor, it estimates the average distance using the locations of both anchors and the hop-count, and sends this back to the network as a correction factor. Upon receiving the correction factor, a blind node is able to estimate its distance to anchors and performs trilateration to estimate its location.

Infrastructure-less schemes generally provide lower accuracy than infrastructure-based schemes. However, by incorporating some range information into the position calculation, the accuracy can be significantly improved. Moreover, due to the merits of distributed computation, low power consumption, low costs and no extra requirements on hardware, infrastructure-less schemes have attracted much attention and research efforts [11], [12], [13], and they have also been exploited in some practical location systems such as Cricket from MIT [14].

\section{System REQUiREMENTS AND DESIGN ISSUES}

This work aims to develop a tracking system which supports continuous, ubiquitous cargo-level tracking. There are a number of technical challenges in developing and deploying such tracking systems. In this section, we first highlight the system design requirements, and then discuss the major design issues.

\section{A. System requirements}

The tracking system requires the cargos to be constantly tracked from a central server. To realize it, each cargo is attached with a tiny battery-powered tracking device, which senses the environment and collects data. Through the communication network, the collected data is transmitted to a central server for monitoring, positioning, and tracking. The detailed requirements are listed as follows.

- Coverage: Global, indoors and outdoors.

- Accuracy: GPS-level accuracy; capable of supporting higher accuracy for areas of special interests (with extra costs).

- Sensing: Besides the positioning feature, the system is expected to be capable of environment sensing, which can be exploited for other applications, such as cool-chain. 
- Duty cycle: On considering the costs and battery life, the location update rate is generally in the order of tens of seconds when cargos are in motion. The rate should be adjustable. Data logging is also required as an option and backup.

- Cost effectiveness: Low deployment cost, low operation and maintenance cost. The battery-powered tracking devices are expected to work for at least a month on average duty cycle.

\section{B. System design issues}

The above system requirements raise a few issues which need to be addressed in the system design. Analyses of these issues lead to the principles of the system design.

1) Positioning indoors and outdoors: The cargo-tracking system envisaged here supports positioning both indoors and outdoors. On considering the characteristics, especially the coverage and availability of various positioning technologies, we focus on GPS and RF (WiFi, ZigBee, RFID, GSM/3G) schemes to build a seamlessly integrated hybrid system. Other technologies, based on infrared [2] or ultrasound [4], [14], will not be explored further as they are not robust for outdoor positioning and only support very limited coverage.

2) Infrastructure-based systems and infrastructure-less systems: As described in Sec. II, infrastructure-less schemes are generally employed for ad hoc networks, especially when a large number of nodes are located in an immediate neighborhood. Such scenario is exactly what can be observed in a cargo-level tracking application, in which many cargos are placed at close proximity to each other. In such a scenario, it is preferred to estimate the relative positioning of cargos by exploiting infrastructure-less positioning method, such as DV-hop [11], [12]. On the other hand, the absolute positions of the anchors can be estimated through some infrastructurebased positioning techniques, such as GPS and WiFi-based system. So our approach will be to estimate the absolute position of all cargos by exploiting both infrastructure-based and infrastructure-less schemes.

3) Public systems and private systems: GPS provides public accessibility with a large coverage outdoors. Since our WiFi-based positioning can work in a fully passive mode by listening to the beacons periodically broadcasted by APs, all existing WiFi APs, no matter public or private, can be exploited as anchor nodes for positioning. From the enduser point of view, utilizing existing infrastructure systems decreases the deployment costs, and thus should be exploited as much as possible as long as sufficient coverage and accuracy can be provided. Meanwhile, private systems, such as WiFi and ZigBee networks, can be deployed as a complement to further improve the total coverage and/or improve the accuracy of some areas of special interests.

4) Realtime data reporting versus data logging: If a communication channel is available, the system can report the collected data to the central server as the data is collected, and thus realtime location tracking can be realized. In case realtime reporting is not necessary or no accessible communication channel is available, the system can also work in data logging mode. The sensed data, for positioning or environmental monitoring, will be stored in the tracking device for post-processing

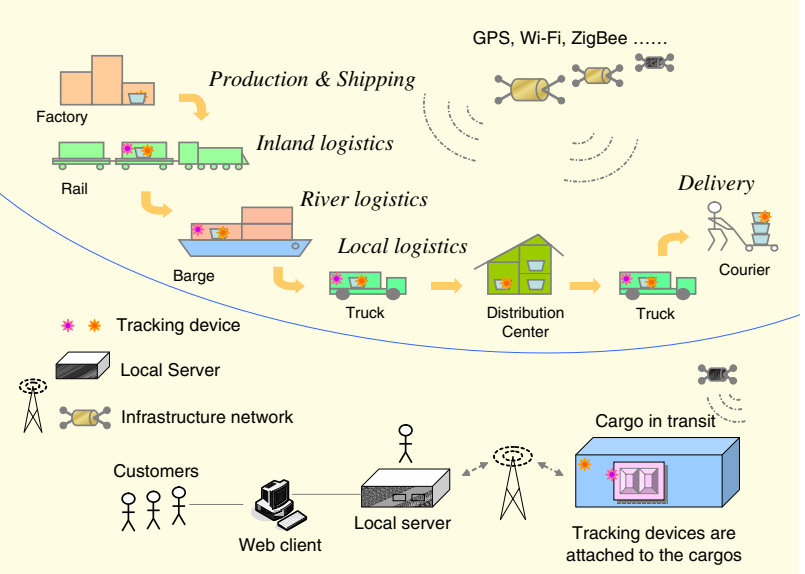

Fig. 1. System architecture of a cargo-level tracking system.

and analysis. Delayed reporting may also be performed when a communication channel is available. The data-logging mode can also be used concurrently with the realtime updating mode as a backup.

5) Wireless data communications: In the proposed system, two kinds of wireless communications are involved. One is for data exchanging between tracking devices and infrastructures. GPRS/3G, WiFi and ZigBee are candidates for this kind of communications. Another kind of communications are ad hoc communications between tracking devices for infrastructureless positioning. Although $\mathrm{WiFi}$ and $\mathrm{ZigBee}$ can both be exploited for ad hoc communications, on considering the data rate, power consumption, and hardware cost, ZigBee is preferred.

6) System costs: System costs include the costs of system manufacturing, deployment, maintenance, and operation. A lower total cost helps to promote the system. Manufacturing costs are related to the hardware configuration of the tracking devices. Deployment costs include the costs of building private infrastructure networks, and the costs for location fingerprint collection, such as wardriving. Costs for maintenance and operation include those to keep the system running properly and smoothly, for example, costs for GSM/3G and WiFi connections, batteries, etc. The above costs may affect each other. For example, a private WiFi network increases the system deployment cost, but decreases the operation cost. Basically, we need to take the working scenario of the specific application into consideration to achieve a good balance.

\section{SYSTEM DESIGN}

\section{A. System overview}

Considering all the design issues as described in Sec. III, we propose a hybrid system to realize all the system requirements. The architecture of the proposed system is illustrated in Fig. 1. The system consists of three key elements:

- Hybrid infrastructure network

- Intelligent tracking device

- Backend central server

Hybrid infrastructure network provides infrastructures for data transmission and positioning. Based on sensing and measurement, tracking devices obtain the information used for position 
calculation and environmental sensing, and send the information to the backend server also via the hybrid infrastructure network. The backend server then calculates the position of the tracking devices and reports to the tracking applications.

In this work, we do not require new infrastructure networks. Instead, we intend to exploit the existing infrastructure networks intelligently. We first introduce our proposed hybrid system and then introduce the intelligent tracking devices and central server in detail.

\section{B. Hybrid system}

The hybrid system proposed in this work involves two kinds of hybrids:

- Hybrid wireless positioning and communication system: Diverse wireless systems are exploited in a seamless and autonomous way. Specifically, GPS, WiFi, ZigBee, and RFID are exploited for positioning, while GSM/3G, $\mathrm{WiFi}$, and ZigBee are utilized for communications. Such hybrid system provides the best service availability and capability.

- Hybrid infrastructure-based and infrastructure-less positioning technology: Two kinds of positioning technologies are exploited in a hybrid and complementary mode to optimize the total deployment costs and operation costs.

In practice, two kinds of tracking devices are deployed in the hybrid system. The first kind is "full-function", and equipped with GPS receiver, transceivers to access WiFi and ZigBee networks, and an RFID tag. Full-function devices support infrastructure-based positioning mechanisms, like GPS and WiFi/ZigBee fingerprint, and can thus be used as anchor nodes in infrastructure-less positioning. The second kind corresponds to "dummy" devices, which are only equipped with low-cost ZigBee transceivers. Dummy devices only support infrastructure-less positioning, and thus can only be tracked with the assistance of full-function devices.

A full-function device costs more than a dummy device. Ideally the fraction of full-function devices should be as low as possible to minimize the total costs. In a practical system, a higher fraction may be adopted to achieve better accuracy and redundancy. The selection of the fraction should also consider the specific requirements of a logistic application.

The full-function tracking devices can also work as dummy devices to save power. The idea is to utilize powerconserving infrastructure-less positioning instead of powerhungry infrastructure-based positioning. Another function of the full-function tracking devices is to perform online fingerprint collection, which serves to complement the wardriving performed in the system deployment stage. By updating the fingerprints of existing anchor nodes and incorporating those of the newly-deployed ones, the online fingerprint collection can continuously improve the accuracy of the system with no extra cost.

To summarize, with the hybrid system design, the average bill-of-materials (BOM) cost of the tracking devices is decreased and the wardriving for system deployment is minimized, thus lowering the system deployment cost. The power consumption of the tracking devices is also decreased, thus lowering the operation cost. Therefore, the return on

\begin{tabular}{c|c|c|c}
\hline & $\begin{array}{c}\text { Infrastructure } \\
\text {-based }\end{array}$ & $\begin{array}{c}\text { Infrastructure } \\
\text {-less }\end{array}$ & Hybrid \\
\hline \hline Position & $\begin{array}{c}\text { Absolute \& } \\
\text { Relative }\end{array}$ & Relative & $\begin{array}{c}\text { Absolute \& } \\
\text { Relative }\end{array}$ \\
\hline Coverage & $\begin{array}{c}\text { Wide } \\
\text { Outdoor/Indoor }\end{array}$ & Limited & $\begin{array}{c}\text { Wide } \\
\text { Outdoor/Indoor }\end{array}$ \\
\hline Scalability & High & Low & High \\
\hline Cost & High & Low & Low \\
\hline $\begin{array}{c}\text { Power } \\
\text { Consumption }\end{array}$ & High & Low & Low \\
\hline
\end{tabular}

TABLE I

COMPARISON OF DIFFERENT POSITIONING TECHNOLOGIES.

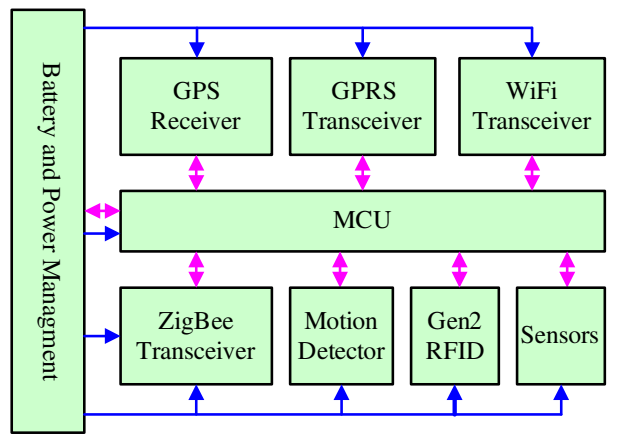

Fig. 2. Block diagram of a tracking device.

investment (ROI) of the end-user is greatly improved. In Table I, we compare the proposed system with infrastructurebased (using WiFi-based positioning as an example) and infrastructure-less tracking technologies to demonstrate the merits of the hybrid solution.

\section{Intelligent tracking device}

We now take a closer look at the tracking device design, focusing on modular design and low power consumption design.

1) Modular design: To achieve enhanced flexibility, the tracking devices employ a "Lego-style" modular design. The block diagram of a full-function tracking device is illustrated in Fig. 2. The major building blocks include: multiple RF interfaces for accessing diverse infrastructure networks, a core micro-controller unit (MCU), a battery module with power management function and a motion detector.

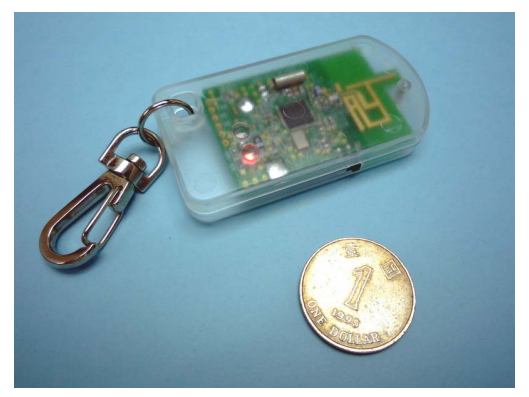

Fig. 3. Prototype of a tracking device. 


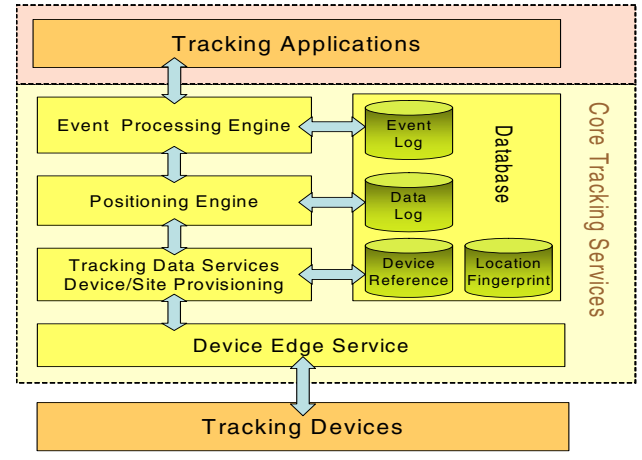

Fig. 4. Architecture of the backend central server.

With the modular design, a dummy tracking device can be built by simply removing some modules from a full-function one. A prototype of a dummy tracking device is shown in Fig. 3. Furthermore, other application-specific tracking devices can also be built easily and rapidly. For instance, WiFi and RFID modules can be snapped together for an indoor tracker; whereas GPS, GPRS modules can also be easily put together for an asset management application which focuses on outdoor rural areas.

2) Low power consumption design: The tracking device is battery-powered and is expected to work for weeks or even months. A long battery life will lower the operation cost of the system. Two kinds of methods are exploited to optimize the power consumption.

- Power conservation in infrastructure-based positioning and communications: Different positioning and communication methods have different power consumption and efficiency. For example, GPS receiver generally consumes less power than a WiFi module. However, GPS needs a few minutes from a cold start, while WiFi-based scheme only requires a few seconds. In data transmissions, GPRS consumes less power than WiFi. But when you consider the average power consumption of each data bit, GPRS consumes more.

- Power conservation by infrastructure-less positioning: As stated above, tracking devices can work in dummy mode to save power by utilizing powerconserving infrastructure-less positioning instead of power-consuming GPS and WiFi-based positioning.

In addition, motion is generally a trigger of position updating. With the motion detector, the tracking device only updates its positioning when motion is detected.

\section{Central server}

Backend central server is another core component of the whole system and is where the tracking applications are located. The central server has a layered structure as shown in Fig. 4. As illustrated in the figure, core tracking services provide all the related services for the development of smart logistic tracking applications to meet the requirements of different logistic users. The features of the major services are briefly introduced below.

- Device Edge Service: Provides interface between tracking devices and central server.
- Tracking Data Services: Perform site and device provisioning and maintenance-related information. Device provisioning is used by the system administrator to register and process the devices before deployment in applications. Site provisioning is used for the location fingerprint generation, maintenance, and updating. The fingerprint includes RF signal information from different positioning technologies, collected through wardriving (during system deployment) and/or online fingerprint collection.

- Positioning Engine: Makes use of fingerprint information (captured by site provisioning) and realtime signals collected by the tracking devices to derive the absolute location and condition of the cargos.

- Event Processing Engine: Interprets and transforms raw tracking data into events and information that supports the application scenarios.

\section{CONCLUSions}

In this paper, we highlight the major requirements of a cargo-level tracking system, and discuss the major design issues involved. Based on the discussion, a hybrid system which incorporates diverse kinds of positioning and communication technologies, and infrastructure-based and infrastructure-less positioning techniques, are proposed. The proposed system achieves enhanced availability, higher accuracy, and lower total costs than existing systems.

\section{REFERENCES}

[1] A. Lamarca et al., "Place lab: Device positioning using radio beacons in the wild," in Proc. of the Third International Conference on Pervasive Computing, May 2005.

[2] R. Want, A. Hopper, V. Falcao, and J. Gibbons, "The active badge location system," ACM Transactions on Information Systems, vol. 10, no. 1, pp. 91-102, 1992.

[3] L. Girod and D. Estrin, "Robust range estimation using acoustic and multimodal sensing," in Proc. of the IEEE/RSJ Int'l Conf. on Intelligent Robots and Systems (IROS 01), vol. 3, 2001, pp. 1312-1320.

[4] A. Harter, A. Hopper, P. Steggles, A. Ward, and P. Webster, "The anatomy of a context-aware application," Wireless Networks, vol. 8, pp. 187-197, March 2002.

[5] D. Niculescu and B. Nath, "Ad hoc positioning system (APS) using AOA," Proc. of IEEE INFOCOM, vol. 3, pp. 1734-1743, Mar. 2003.

[6] G. Carter, "Coherence and time delay estimation," Proceedings of the IEEE, vol. 75, no. 2, pp. 236-255, Feb. 1987.

[7] K. Pahlavan, P. Krishnamurthy, and A. Beneat, "Wideband radio propagation modeling for indoor geolocation applications," IEEE Communications Magazine, vol. 36, no. 4, p. 60šC65, 1998.

[8] J. Hightower and G. Borriello, "Location systems for ubiquitous computing," IEEE Computer, vol. 34, no. 8, pp. 57-66, Aug. 2001.

[9] P. Bahl and V. Padmanabhan, "RADAR: An in-building RF-based user location and tracking system," Proc. of IEEE INFOCOM, vol. 2, pp. 775-784, 2000.

[10] M. Y. Chen et al., "Practical metropolitan-scale positioning for GSM phones," in Proc. of UbiComp. Springer, 2006, pp. 225-242.

[11] D. Niculescu and B. Nath, "Ad hoc positioning system (APS)," in Proc. of IEEE Global Telecommunications Conf., vol. 5, San Antonio, November 2001, pp. 2926-2931.

[12] — "DV based positioning in ad hoc networks," Journal of Telecommunication Systems, vol. 22(1/4), pp. 267-280, 2003.

[13] S. Capkun, M. Hamdi, and J.-P. Hubaux, "GPS-free positioning in mobile ad-hoc networks," Cluster Computing, vol. 5(2), no. 157-167, 2002.

[14] N. Priyantha, A. Chakraborty, and H. Balakrishnan, "The cricket location-support system," in Proc. of 6th Annual Int'l Conf. on Mobile Computing and Networking, 2000, pp. 32-43. 